\title{
Matchings, cycle bases, and the maximum genus of a graph
}

\author{
Michal Kotrbčík Martin Škoviera \\ Department of Computer Science \\ Faculty of Mathematics, Physics, and Informatics \\ Comenius University \\ Bratislava, Slovakia \\ \{kotrbcik, skoviera\}@dcs.fmph.uniba.sk
}

Submitted: Mar 31, 2011; Accepted: Jun 26, 2012; Published: Jul 5, 2012

Mathematics Subject Classifications: 05C10, 05C38, 05C70

\begin{abstract}
We study the interplay between the maximum genus of a graph and bases of its cycle space via the corresponding intersection graph. Our main results show that the matching number of the intersection graph is independent of the basis precisely when the graph is upper-embeddable, and completely describe the range of matching numbers when the graph is not upper-embeddable. Particular attention is paid to cycle bases consisting of fundamental cycles with respect to a given spanning tree. For 4-edge-connected graphs, the intersection graph with respect to any spanning tree (and, in fact, with respect to any basis) has either a perfect matching or a matching missing exactly one vertex. We show that if a graph is not 4-edge-connected, different spanning trees may lead to intersection graphs with different matching numbers. We also show that there exist 2-edge-connected graphs for which the set of values of matching numbers of their intersection graphs contains arbitrarily large gaps.
\end{abstract}

Keywords: maximum genus; matching; cycle space; fundamental cycle

\section{Introduction}

The maximum genus of a graph $G, \gamma_{M}(G)$, is the largest genus of an orientable surface upon which $G$ has a 2-cell embedding. This concept was introduced by Nordhaus, Stewart, and White [13] in 1971, and immediately attracted considerable attention; a survey of an early research in this area can be found in [15]. At present, maximum genus seems to be fairly well understood, especially because it admits a so-called good characterization in purely combinatorial terms $[9,8,11,17]$ and can be computed in a polynomial time. 
In fact, two essentially different polynomial-time algorithms are known, one by Glukhov [4] and another one by Furst, Gross, and McGeoch [2]. A third algorithm, based on intersection properties of fundamental cycles, has been recently proposed by Ren, Zhang, and $\mathrm{Li}$ in [14]. Intersection graphs of fundamental cycles, and more generally, of cycle bases of a graph, have already been used to characterise the maximum genus, see [5], [12], and [3]. However, matching properties of these graphs remain largely unknown and therefore deserve further investigation.

Recall that the fundamental cycle $T(e)$ with respect to a spanning tree $T$ of a graph $G$ is the only cycle that passes trough a cotree edge $e$ and is contained in $T+e$. The idea of using fundamental cycles to study the maximum genus has a fairly long history: Glukhov [5] and Nebeský [12] determined the maximum genus of a graph by considering the intersection graphs of bases of its cycle space. Given a graph $G$ and a basis $\mathcal{B}$ of its cycle space, one can define the graph $J(G, \mathcal{B})$ whose vertices are the elements of $\mathcal{B}$ and edges join pairs of elements that have a vertex in common. A particularly important case arises when the basis $\mathcal{B}_{T}$ consisting of fundamental cycles with respect to a spanning tree $T$ is chosen. In this situation the intersection graph $J\left(G, \mathcal{B}_{T}\right)$ is simply denoted by $G \sharp T$. The fundamental property of these graphs is that $\gamma_{M}(G)$ can be expressed through their matching numbers. The following theorem of Fu, Škoviera, and Tsai [3] is a common generalization of results from [5] and [12]. It is stated here in a slightly modified form, with $\nu(H)$ denoting the matching number of a graph $H$, the size of a maximum matching in $H$.

Theorem 1. Let $G$ be a connected graph. Then

$$
\gamma_{M}(G)=\min \nu(J(G, \mathcal{B}))
$$

where the minimum is taken over all bases $\mathcal{B}$ of the cycle space of $G$. Furthermore, there is a spanning tree $T$ such that

$$
\gamma_{M}(G)=\nu(G \sharp T) .
$$

The purpose of this paper is to reveal a rather unexpected behaviour of the matching number $\nu(J(G, \mathcal{B}))$ for different bases of the cycle space of $G$. We show in Theorem 2 that this matching number does not depend on the chosen basis precisely when the graph is upper-embeddable, that is, when $G$ admits a 2-cell embedding with at most two faces. In Theorem 3 we describe the complete range of matching numbers of the intersection graphs $J(G, \mathcal{B})$ : we prove that $G$ has a basis $\mathcal{B}$ with $\nu(J(G, \mathcal{B}))=k$ if and only if $\gamma_{M}(G) \leqslant k \leqslant\lfloor\beta(G) / 2\rfloor$, where $\beta(G)$ is the cycle rank of $G$.

The situation dramatically changes when we restrict to cycle bases consisting of fundamental cycles. The analogue of Theorem 2 is no more valid because, together with the upper-embeddable graphs, there also exist certain non-upper-embeddable graphs $G$ for which the matching number $\nu(G \sharp T)$ does not depend on the chosen spanning tree. At the same time, there are graphs $G$ such that $\nu(G \sharp T)$ does depend on the choice of the spanning tree. The edge connectivity of such graphs is necessarily bounded above by 3 since every 4 -edge-connected graph is upper-embeddable $[7,18]$. We display an infinite family of 2-edge-connected graphs $\left(G_{n}\right)_{n \geqslant 1}$ such that each $G_{n}$ contains spanning 
trees $T_{1}$ and $T_{2}$ with $\nu\left(G_{n} \sharp T_{1}\right)-\nu\left(G_{n} \sharp T_{2}\right)=n$ and any other spanning tree $T$ has either $\nu\left(G_{n} \sharp T\right)=\nu\left(G_{n} \sharp T_{1}\right)$ or $\nu\left(G_{n} \sharp T\right)=\nu\left(G_{n} \sharp T_{2}\right)$. In other words, the range of values of $\nu\left(G_{n} \sharp T\right)$ contains a gap of size $n$; such a gap cannot occur if general cycle bases are considered. We further construct an infinite family of 3-edge-connected graphs $\left(H_{n}\right)_{n \geqslant 1}$ such that each $H_{n}$ contains spanning trees $T_{1}$ and $T_{2}$ with $\nu\left(H_{n} \sharp T_{1}\right)-\nu\left(H_{n} \sharp T_{2}\right)=n$. Whether there exist 3-edge-connected graphs with arbitrarily large gaps in the range of the values of $\nu(G \sharp T)$ remains open.

The maximum genus algorithm from [14] uses the assumption that $\nu(G \sharp T)=\gamma_{M}(G)$ for every spanning tree $T$ of an arbitrary graph $G$ and determines $\gamma_{M}(G)$ by randomly choosing $T$ and computing the value of $\nu(G \sharp T)$. As follows from our results, for graphs with small edge-connectivity this approach fails.

\section{Preliminaries}

In the next few paragraphs we collect several basic definitions needed in this paper. A circuit in a graph $G$ is a connected regular subgraph of valency two. A cycle in $G$ is a spanning subgraph having all vertices of even valency. The set $\mathcal{C}(G)$ of all cycles of $G$ forms a vector space over the 2-element field $G F(2)$ with sum $C_{1}+C_{2}$ defined as the symmetric difference of the edge-sets of cycles $C_{1}$ and $C_{2}$ and with scalar multiplication $0 \cdot C=0$ and $1 \cdot C=C$. A cycle basis of $G$ is any basis of $\mathcal{C}(G)$. The size of any cycle basis is denoted by $\beta(G)$ and is called the Betti number or the cycle rank of $G$. Recall that every spanning tree $T$ in a connected graph $G$ gives rise to a cycle basis $\mathcal{B}_{T}$ consisting of all fundamental cycles with respect to $T$. It follows that, for connected graphs, $\beta(G)=|E(G)|-|V(G)|+1$.

Throughout this paper the reader is assumed to be familiar with fundamentals of topological graph theory as presented, for example, in the monograph of Gross and Tucker [6]. We recall that the maximum genus $\gamma_{M}(G)$ of a connected graph $G$ is the largest genus among those orientable surfaces into which $G$ has a 2-cell embedding. It is well known that the maximum genus of every graph $G$ satisfies the inequality $\gamma_{M}(G) \leqslant\lfloor\beta(G) / 2\rfloor$. A graph for which the equality holds is called upper-embeddable.

It is often convenient to express the maximum genus of a graph $G$ through an equivalent quantity, its Betti deficiency $\xi(G)$. This is defined by setting

$$
\xi(G)=\beta(G)-2 \gamma_{M}(G)
$$

Betti deficiency has two complementary combinatorial characterisations. The first of them is a theorem of Xuong [17] stating that for every connected graph $G$ one has

$$
\xi(G)=\min \xi(G, T)
$$

where $\xi(G, T)$ denotes the number of components of the cotree $G-E(T)$ with odd number of edges and the minimum is taken over all spanning trees $T$ of $G$. A spanning tree reaching this minimum is called a Xuong tree. Every Xuong tree also attains the minimum of 
formula (1) in Theorem 1 (see for example [1]) but not necessarily vice versa. Examples of graphs with a spanning tree that satisfies (1) but is not a Xuong tree are easy to find.

The second characterisation requires a little more terminology. Recall that a leaf of a graph is a 2-edge-connected subgraph maximal under inclusion. A leaf is cyclically even if its Betti number is even, and is cyclically odd otherwise. The following result was proved by Khomenko and Glukhov [8] and independently, though in a slightly different form, by Nebeský [11]: For every connected graph $G$ one has

$$
\xi(G)=\max \mathrm{ol}(G-A)-|A|
$$

where ol $(H)$ denotes the number of cyclically odd leaves of a graph $H$ and the maximum is taken over all subsets $A \subseteq E(G)$. An accessible proof of this formula can be found in [16, Theorem 2] within a broader context of signed graphs.

\section{Matchings in cycle spaces}

The following theorem is formally one of many characterisations of upper-embeddable graphs, but in fact it is a result about cycle spaces of graphs. It characterises graphs in which the intersection graphs of any two cycle bases have the same matching number. Surprisingly, this class coincides with the class of all upper-embeddable graphs.

Theorem 2. A connected graph $G$ is upper-embeddable if and only if the intersection graphs of any two cycle bases have the same matching number. Furthermore, if $G$ is upper-embeddable, then the matching number coincides with $\gamma_{M}(G)=\lfloor\beta(G) / 2\rfloor$.

Proof. Assume that $G$ is upper-embeddable. Theorem 1 implies that $\min _{\mathcal{B}} \nu(J(G, \mathcal{B}))=$ $\gamma_{M}(G)=\lfloor\beta(G) / 2\rfloor$, where the minimum is taken over all bases $\mathcal{B}$ of the cycle space of $G$. Hence, for an arbitrary fixed cycle basis $\mathcal{D}$ of $G$ we get

$$
\nu(J(G, \mathcal{D})) \geqslant \min _{\mathcal{B}}(J(G, \mathcal{B}))=\lfloor\beta(G) / 2\rfloor .
$$

On the other hand, the graph $J(G, \mathcal{D})$ has $\beta(G)$ vertices, and therefore

$$
\nu(J(G, \mathcal{D})) \leqslant\lfloor\beta(G) / 2\rfloor .
$$

It follows that $\nu(J(G, \mathcal{D}))=\lfloor\beta(G) / 2\rfloor$ for every cycle basis $\mathcal{D}$ of $G$ whenever $G$ is upperembeddable, as claimed.

For the converse assume that $G$ is not upper-embeddable. We intend to show that $G$ has two cycle bases $\mathcal{B}_{1}$ and $\mathcal{B}_{2}$ such that $\nu\left(J\left(G, \mathcal{B}_{1}\right)\right) \neq \nu\left(J\left(G, \mathcal{B}_{2}\right)\right)$. Let $\mathcal{B}_{1}$ be the basis $\mathcal{B}_{T}$ consisting of the fundamental cycles with respect to a Xuong tree $T$ of $G$ (or with respect to any spanning tree $T$ of $G$ that attains the minimum in Theorem 1 ). It follows that $\nu(G \sharp T)=\gamma_{M}(G)$. Take an arbitrary maximum matching $M$ of $G \sharp T$ and choose any two vertices $u$ and $v$ of $G \sharp T$ that are not matched by $M$; the existence of such vertices follows from the fact that $G$ is not upper-embeddable. Since $M$ is a maximal matching, $u$ 
and $v$ are not adjacent in $G \sharp T$. Recall that $u$ and $v$ are fundamental cycles with respect to $T$, so there exist cotree edges $e$ and $f$ of $G$ such that $u=T(e)$ and $v=T(f)$. Define the set $\mathcal{B}_{2}$ by setting $\mathcal{B}_{2}=\left(\mathcal{B}_{1}-\{T(f)\}\right) \cup\{T(e)+T(f)\}$. It is easy to see that $\mathcal{B}_{2}$ is again a cycle basis and that $J\left(G, \mathcal{B}_{1}\right)$ is isomorphic to a subgraph of $J\left(G, \mathcal{B}_{2}\right)$ via the injective homomorphism $\phi: J\left(G, \mathcal{B}_{1}\right) \rightarrow J\left(G, \mathcal{B}_{2}\right)$ which maps $T(f)$ to $T(e)+T(f)$ and otherwise sends an element $C \in \mathcal{B}_{1}$ to $C \in \mathcal{B}_{2}$. The image $\phi(M)$ of the matching $M$ is a matching in $J\left(G, \mathcal{B}_{2}\right)$. Since $T(e)$ and $T(e)+T(f)=\phi(T(f))$ have a nonempty intersection, they are adjacent as vertices of $J\left(G, \mathcal{B}_{2}\right)$. Thus we can extend the matching $\phi(M)$ of $J\left(G, \mathcal{B}_{2}\right)$ with the edge joining $T(e)$ to $T(e)+T(f)$, thereby obtaining a larger matching $M^{\prime}$. Consequently,

$$
\nu\left(J\left(G, \mathcal{B}_{2}\right)\right) \geqslant\left|M^{\prime}\right|>|M|=\nu\left(J\left(G, \mathcal{B}_{1}\right)\right),
$$

and the result follows.

Our next result strenghtens Theorem 2 and provides a complete description of the matching numbers of intersection graphs $J(G, \mathcal{B})$ for different cycle bases of a graph $G$. In its proof we use several concepts from matching theory; for details we refer the reader to Lovász and Plummer [10]. A connected component of a graph is called odd if it has an odd number of vertices; the number of odd components of a graph $H$ is denoted by $\omega(H)$. The matching deficiency of a graph $H$, denoted $\theta(H)$, is the number of vertices not covered by a maximum matching of $H$, that is, $\theta(H)=|V(G)|-2 \nu(H)$. The Berge Formula states that the matching deficiency of an arbitrary graph $G$ satisfies the equation

$$
\theta(G)=\max \{\omega(G-X)-|X|\}
$$

where the maximum is taken over all sets $X \subseteq V(G)$. If $v_{1}, v_{2}, \ldots, v_{n}$ is the set of vertices not covered by a maximum matching of $G$ and a set $X \subseteq V(G)$ satisfies $\theta(G)=$ $\omega(G-X)-|X|$, then the vertices $v_{i}$ belong to different odd components of $G-X$. The Berge Formula and its proof can be found in [10].

Theorem 3. Let $G$ be a connected graph. Then $G$ has a cycle basis $\mathcal{B}$ with $\nu(J(G, \mathcal{B}))=k$ if and only if $\gamma_{M}(G) \leqslant k \leqslant\lfloor\beta(G) / 2\rfloor$.

Proof. If $\mathcal{B}$ is a cycle basis with $\nu(J(G, \mathcal{B}))=k$, then $k \geqslant \gamma_{M}(G)$ by Theorem 1 , and $k \leqslant$ $\lfloor\beta(G) / 2\rfloor$, because $J(G, \mathcal{B})$ has $\beta(G)$ vertices. This establishes the necessary condition of the theorem.

To prove the sufficiency, we construct, for every integer $k$ with $\gamma_{M}(G) \leqslant k \leqslant\lfloor\beta(G) / 2\rfloor$, a basis $\mathcal{B}$ such that $\nu(J(G, \mathcal{B}))=k$. Assume that the graph $G$ has Betti deficiency $\xi(G)=d$. Recall that by $(2)$ we have $d=\beta(G)-2 \gamma_{M}(G)$. We start our construction with a basis $\mathcal{B}_{T}$ where $T$ is a spanning tree of $G$ that attains the minimum in Theorem 1; obviously, $\nu\left(J\left(G, \mathcal{B}_{T}\right)\right)=\gamma_{M}(G)$. Take an arbitrary maximum matching $M$ of $J\left(G, \mathcal{B}_{T}\right)$ and note that $M$ leaves precisely $d$ vertices unmatched. Consequently, $d=\theta(J(G, \mathcal{B})$ ). Denote the unmatched vertices by $v_{1}, v_{2}, \ldots, v_{d}$. As each $v_{j}$ is a fundamental cycle with respect to $T$, there exists a cotree edge $e_{j}$ of $G$ such that $v_{j}=T\left(e_{j}\right)$. Let $\mathcal{B}_{0}=\mathcal{B}_{T}$ and let

$$
\mathcal{B}_{i}=\mathcal{B}_{i-1}-\left\{T\left(e_{2 i}\right)\right\} \cup\left\{T\left(e_{2 i-1}\right)+T\left(e_{2 i}\right)\right\}
$$


for $i=1,2, \ldots,\lfloor d / 2\rfloor$. Each $\mathcal{B}_{i}$ is obviously a basis of the cycle space of $G$.

As in the proof of Theorem 2, it is easy to see that $J\left(G, \mathcal{B}_{i-1}\right)$ is isomorphic to a subgraph of $J\left(G, \mathcal{B}_{i}\right)$ via the injective homomorphism that sends the vertex $T\left(e_{2 i}\right)$ to $T\left(e_{2 i-1}\right)+T\left(e_{2 i}\right)$ and maps all other vertices identically. In the rest of the proof we will slightly abuse the notation by identifying the vertices of $J\left(G, \mathcal{B}_{i-1}\right)$ with those in $J\left(G, \mathcal{B}_{i}\right)$ according to this injective homomorphism. As the vertex $T\left(e_{2 i}\right)$ in $J\left(G, \mathcal{B}_{i-1}\right)$ and the vertex $T\left(e_{2 i-1}\right)+T\left(e_{2 i}\right)$ in $J\left(G, \mathcal{B}_{i}\right)$ are the only vertices where $J\left(G, \mathcal{B}_{i-1}\right)$ and $J\left(G, \mathcal{B}_{i}\right)$ differ, no confusion can occur.

Our aim is to show that $\nu\left(J\left(G, \mathcal{B}_{i}\right)\right)=\gamma_{M}(G)+i$ for each $i \leqslant\lfloor d / 2\rfloor$. Clearly, it suffices to prove the following claim.

\section{Claim.}

(a) The graph $J\left(G, \mathcal{B}_{i}\right)$ has $\theta\left(J\left(G, \mathcal{B}_{i}\right)\right)=d-2 i$ for each $i \geqslant 0$.

(b) The graph $J\left(G, \mathcal{B}_{i}\right)$ with $i \leqslant\lfloor d / 2\rfloor-1$ has a maximum matching $M_{i}$ that leaves unmatched exactly the vertices $v_{2 i+1}, v_{2 i+2}, \ldots, v_{d}$.

We proceed by induction on $i$. The case $i=0$ follows from the choice of $T$ by putting $M_{0}=M$. Assume that $i>0$. From the induction hypothesis and the Berge Formula (5) we get that there is a set $X \subseteq V\left(J\left(G, \mathcal{B}_{i-1}\right)\right)$ such that $J\left(G, \mathcal{B}_{i-1}\right)-X$ has precisely $\theta\left(J\left(G, \mathcal{B}_{i-1}\right)\right)+|X|$ odd components, and the vertices $v_{2 i-1}, v_{2 i}, \ldots, v_{d}$ belong to different odd components of $J\left(G, \mathcal{B}_{i-1}\right)-X$. The definition of $\mathcal{B}_{i}$ implies that $v_{2 i-1}$ and $v_{2 i}$ are joined by an edge in $J\left(G, \mathcal{B}_{i}\right)$ and therefore we can set $M_{i}=M_{i-1} \cup\left\{v_{2 i-1} v_{2 i}\right\}$. Clearly, the vertices $v_{2 i+1}, v_{2 i+2}, \ldots, v_{d}$ remain unmatched by $M_{i}$.

Now we prove that $J\left(G, \mathcal{B}_{i}\right)$ does not contain a matching larger than $M_{i}$, that is, $\theta\left(J\left(G, \mathcal{B}_{i}\right)\right)=\theta\left(J\left(G, \mathcal{B}_{i-1}\right)\right)-2$. Since $M_{i}$ leaves $\theta\left(J\left(G, \mathcal{B}_{i-1}\right)-2\right.$ vertices uncovered, we obtain $\theta\left(J\left(G, \mathcal{B}_{i}\right)\right) \leqslant \theta\left(J\left(G, \mathcal{B}_{i-1}\right)\right)-2$. On the other hand, the Berge Formula (5) yields that

$$
\theta\left(J\left(G, \mathcal{B}_{i}\right)\right)=\max _{Y \subseteq V\left(J\left(G, \mathcal{B}_{i}\right)\right)} \omega\left(J\left(G, \mathcal{B}_{i}\right)\right)-|Y| .
$$

Notice that the difference between graphs $J\left(G, \mathcal{B}_{i-1}\right)$ and $J\left(G, \mathcal{B}_{i}\right)$ is only at the vertex $v_{2 i}$ : its neighbourhood in $J\left(G, \mathcal{B}_{i}\right)$ is the union of the neighbourhoods of $v_{2 i-1}$ and $v_{2 i}$ in $J\left(G, \mathcal{B}_{i-1}\right)$. By the induction hypothesis the vertices $v_{2 i-1}$ and $v_{2 i}$ belong to different odd components of $J\left(G, \mathcal{B}_{i-1}\right)-X$. It follows that the graph $J\left(G, \mathcal{B}_{i}\right)-|X|$ has $\theta\left(J\left(G, \mathcal{B}_{i-1}\right)\right)+$ $|X|-2$ odd components. Therefore

$$
\begin{aligned}
\theta\left(J\left(G, \mathcal{B}_{i}\right)\right) & =\max _{Y \subseteq V\left(J\left(G, \mathcal{B}_{i}\right)\right)}\left\{\omega\left(J\left(G, \mathcal{B}_{i}\right)\right)-|Y|\right\} \\
& \geqslant \theta\left(J\left(G, \mathcal{B}_{i}\right)\right)-|X|=\theta\left(\left(J\left(G, \mathcal{B}_{i-1}\right)\right)-2 .\right.
\end{aligned}
$$

This completes the induction step as well as the proof of the theorem.

We finish this section by applying Theorem 2 to 4-edge-connected graphs.

Corollary 4. If $G$ is a 4-edge-connected graph, then $\nu(J(G, \mathcal{B}))=\lfloor\beta(G) / 2\rfloor$ irrespectively of the chosen basis $\mathcal{B}$ of the cycle space of $G$.

Proof. Every 4-edge-connected graph is upper-embeddable by a result of Jungerman [7] and Xuong [18]. The conclusion now follows from Theorem 2. 


\section{Matchings of fundamental cycles}

In this section we focus on bases of the cycle space that only consist of fundamental cycles. We show that the properties of intersection graphs of fundamental cycles are in several ways different from those that come from general cycle bases. We start with a corollary of Theorem 2.

Corollary 5. If $G$ is an upper-embeddable graph, then $\nu\left(G \sharp T_{1}\right)=\nu\left(G \sharp T_{2}\right)$ for any two spanning trees $T_{1}$ and $T_{2}$ of $G$.

In view of Theorem 2 it is natural to ask whether this corollary can be reversed. As the next example shows, the answer is 'no'.

Example 6. Let $A_{n}$ be a circuit on $n \geqslant 3$ vertices with a loop attached to every vertex. It is not difficult to see that $\gamma_{M}\left(A_{n}\right)=1$ and that $A_{n}$ is not upper-embeddable. Observe that any two spanning trees $T_{1}$ and $T_{2}$ give rise to the same cycle basis $\mathcal{B}$ which consists of the unique circuit $C$ of length $n$ and $n$ circuits $C_{i}$ for $i=1,2, \ldots, n$ of length 1 corresponding to the loops of $A_{n}$. Hence, any two spanning trees $T_{1}$ and $T_{2}$ of $A_{n}$ give rise to isomorphic intersection graphs $A_{n} \sharp T_{1}$ and $A_{n} \sharp T_{2}$, all of them isomorphic to the complete bipartite graph $K_{1, n}$. Therefore $\nu\left(A_{n} \sharp T_{1}\right)=\nu\left(A_{n} \sharp T_{2}\right)=1$ for any two spanning trees $T_{1}$ and $T_{2}$ of $A_{n}$. This shows that the analogue of Theorem 2 for cycle bases consisting of fundamental cycles is false.

We have seen in Corollary 4 that the intersection graphs of any two cycle bases of a 4edge-connected graphs have the same matching number. We now investigate the situation in 2-edge-connected graphs and 3-edge-connected graphs.

Our first aim is to show that the set of values of the matching numbers $\nu(G \sharp T)$ for a 2-edge-connected graph $G$ may contain gaps of arbitrary size. To construct the required graphs, take a path on $2 n+2$ vertices and, starting from its second edge, replace every other edge with a pair of parallel edges. Let $N_{n}$ denote the resulting graph. Next, join the two pendant vertices of $N_{n}$ with $n$ parallel edges and denote the resulting graph by $L_{n}$. Clearly, $L_{n}$ is 2-edge-connected but is not 3-edge-connected. An example of this construction is shown in Figure 1.
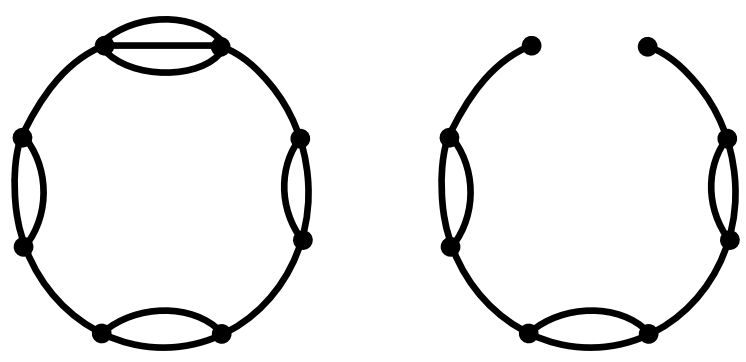

Figure 1: The graph $L_{3}$ (left) and its spanning subgraph $N_{3}$ (right)

We determine the maximum genus of $L_{n}$. 
Proposition 7. The graph $L_{n}$ has $\xi\left(L_{n}\right)=n$ for $n \geqslant 2$ even, and has $\xi\left(L_{n}\right)=n-1$ for $n \geqslant 1$ odd. Consequently, for every positive integer $n$ we have

$$
\gamma_{M}\left(L_{n}\right)=\left\lfloor\frac{n+1}{2}\right\rfloor
$$

Proof. Consider the graph $L_{n}$ for a fixed $n$. Take any two adjacent vertices that are joined by a single edge, denote this edge by $e$, and set $A=\{e\}$. Clearly, $L_{n}-A$ is connected and has either $n+1$ cyclically odd leaves if $n$ is even, or $n$ cyclically odd leaves if $n$ is odd. From (4) we then get that $\xi\left(L_{n}\right) \geqslant \operatorname{ol}\left(L_{n}-A\right)-|A|$, which equals either $n$ for $n$ even, or equals $n-1$ for $n$ odd. On the other hand, for any spanning tree $S$ of $L_{n}-e$ the expression (3) yields that $\xi\left(L_{n}\right) \leqslant \xi\left(L_{n}, S\right)$, which again equals either $n$, when $n$ is even, or equals $n-1$, when $n$ is odd. This establishes the Betti deficiency of $L_{n}$. The formula for the maximum genus of $L_{n}$ follows immediately from (2) and the fact that $\beta\left(L_{n}\right)=2 n$ for every $n \geqslant 1$.

We now turn our attention to matchings in the intersection graphs $L_{n} \sharp T$ of fundamental cycles of the graph $L_{n}$.

Proposition 8. For every positive integer $n$, the graph $L_{n}$ has a spanning tree $U$ such that the graph $L_{n} \sharp U$ has a perfect matching; in particular, $\nu\left(L_{n} \sharp U\right)=n$.

Proof. Choose any spanning tree $U$ of $L_{n}$ within the spanning subgraph $N_{n}$. The fundamental cycles of $L_{n}$ with respect to $U$ can be split into two sets $C$ and $D$ of $n$ elements each in the following way. The set $C$ will include the fundamental cycles corresponding to the edges from $E\left(L_{n}\right)-E\left(N_{n}\right)$ while $D$ will have the rest. Clearly, no two members of $D$ intersect, while every element of $C$ intersects all other elements of $C \cup D$. Thus $L_{n} \sharp U$ is isomorphic to the union of the complete graph $K_{n}$ on the set $C$ with the complete bipartite graph $K_{n, n}$ on the partite sets $C$ and $D$. A perfect matching in $L_{n} \sharp U$ can now be constructed by simply choosing a bijection $C \rightarrow D$ and matching an element of $C$ to its image in $D$.

The following result shows that for a 2-edge-connected graph $G$ not only the range of values of $\nu(G \sharp T)$ can be nontrivial but it can also contain arbitrarily large gaps.

Theorem 9. For every positive integer $n$ there exists a 2-edge-connected graph $G_{n}$ such that

$$
\max _{T} \nu\left(G_{n} \sharp T\right)-\min _{T} \nu\left(G_{n} \sharp T\right)=n
$$

where $T$ ranges over all spanning trees of $G_{n}$. Moreover, for every spanning tree $S$ of $G_{n}$ the value of $\nu\left(G_{n} \sharp S\right)$ coincides with one of the two extremal values.

Proof. For each $n \geqslant 1$ set $G_{n}=L_{2 n}$; clearly, $G_{n}$ is 2-edge-connected. Theorem 1 and Proposition 7 imply that for the spanning tree $S$ described in the proof of Proposition 7 we have

$$
\min _{T} \nu\left(G_{n} \sharp T\right)=\nu\left(L_{2 n} \sharp S\right)=\gamma_{M}\left(L_{2 n}\right)=\lfloor(2 n+1) / 2\rfloor=n .
$$


On the other hand, the spanning tree $U$ from Proposition 8 gives

$$
\max _{T} \nu\left(G_{n} \sharp T\right)=\nu\left(L_{2 n} \sharp U\right)=2 n .
$$

It is not difficult to see that if $T$ is a spanning tree of $L_{2 n}$, then $\nu\left(G_{n} \sharp T\right)=2 n$. On the other hand, if $T$ contains any edge outside $L_{2 n}$, then $\nu(G \sharp T)=n$. The result follows.

We proceed to 3-edge-connected graphs. We intend to show that for any integer $n$ there is a 3-edge-connected graph $H_{n}$ such that $\nu\left(H_{n} \sharp T_{1}\right)-\nu\left(H_{n} \sharp T_{2}\right)=n$ for suitable spanning trees $T_{1}$ and $T_{2}$ of $H_{n}$. Denote by $v_{1}, v_{2}, \ldots, v_{4 n+4}$ the vertices along the longest path of $N_{2 n+1}$, starting from a pendant vertex of $N_{2 n+1}$. For every $i=1,2, \ldots, 2 n+1$, subdivide one of the edges between $v_{2 i}$ and $v_{2 i+1}$ with a vertex $u_{i}$. Join the vertex $u_{i}$ to the vertex $u_{n+1+i}$ for every $i=1,2, \ldots, n$ and join the vertex $u_{n+1}$ to $v_{1}$. Join $v_{1}$ to $v_{4 n+4}$ with $n$ parallel edges and denote the resulting graph by $O_{n}$. It is not difficult to see that the graph $O_{n}$ is 3 -edge-connected. The result of this process for $n=4$ is illustrated in Figure 2.

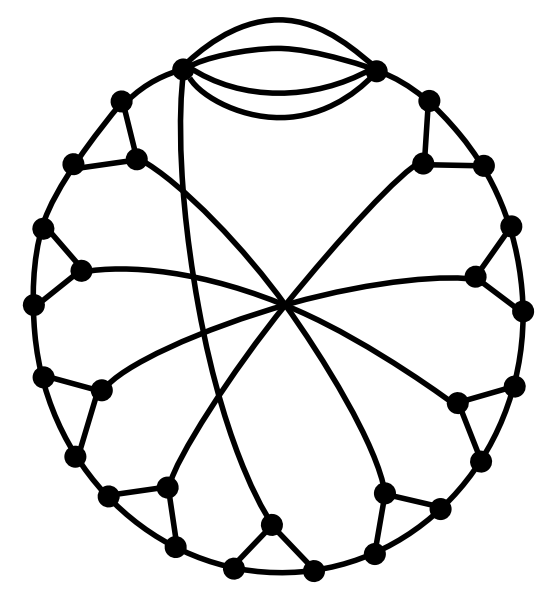

Figure 2: The graph $O_{4}$

Proposition 10. The graph $O_{n}$ has $\xi\left(O_{n}\right)=n$ for $n \geqslant 2$ even, and has $\xi\left(O_{n}\right)=n-1$ for $n \geqslant 1$ odd. Consequently, for every positive integer $n$ we have

$$
\gamma_{M}\left(O_{n}\right)=1+\left\lceil\frac{3 n}{2}\right\rceil
$$

Proof. Consider the graph $O_{n}$ for a fixed $n$. Let $A$ be the set consisting of the edges $v_{1} v_{2}$ and $v_{1} u_{2 n+1}$ and of all edges $u_{i} u_{n+1+i}$ for $1 \leqslant i \leqslant n$. Clearly, $|A|=n+2$. The graph $O_{n}-A$ is connected and has $2 n+2$ cyclically odd leaves if $n$ is even, and $2 n+1$ cyclically odd leaves if $n$ is odd. From (4) we then get that $\xi\left(O_{n}\right) \geqslant \operatorname{ol}\left(O_{n}-A\right)-|A|$, which equals either $n$ for $n$ even, or equals $n-1$ for $n$ odd. Let $X$ be the set of edges of $O_{n}$ constructed as follows. First, include in $X$ all edges of $O_{n}$ of the form $v_{i} v_{i+1}$ for each $1 \leqslant i \leqslant 4 n+4$, with indices 
taken modulo $4 n+4$, except for of the edges $v_{2 n+1} v_{2 n+2}, v_{2 n+2} v_{2 n+3}$, and $v_{2 n+3} v_{2 n+4}$. Add to $X$ the edges $v_{2 i} u_{i}$ for all $1 \leqslant i \leqslant n+1$ and $v_{2 i+1} u_{i}$ for all $n+1 \leqslant i \leqslant 2 n+1$. Clearly, $X$ is a spanning tree of $O_{n}$; for $n=4$ this spanning tree is illustrated in Figure 3 (left). The expression (3) yields that $\xi\left(O_{n}\right) \leqslant \xi\left(O_{n}, X\right)$, which equals either $n$, when $n$ is even, or equals $n-1$, when $n$ is odd. This establishes the Betti deficiency of $O_{n}$. The formula for the maximum genus of $O_{n}$ follows from (2) and the fact that $\beta\left(O_{n}\right)=4 n+2$ for every $n \geqslant 1$.

Proposition 11. For every positive integer $n$, the graph $O_{n}$ has a spanning tree $Y$ such that the graph $O_{n} \sharp Y$ has a perfect matching; in particular, $\nu\left(O_{n} \sharp Y\right)=n$.

Proof. Let $Y=\left\{v_{1} v_{2}, v_{2} v_{3}, \ldots, v_{4 n+3} v_{4 n+4}, v_{2} u_{1}, v_{4} u_{2}, \ldots, v_{4 n+2} u_{2 n+1}\right\}$. Clearly, $Y$ is a spanning tree of $O_{n}$; for $n=4$ this spanning tree is illustrated in Figure 3 (right). Let $A$ be the set consisting of all vertices of $O_{n} \sharp Y$ that correspond to the fundamental cycles containing the edges between $v_{1}$ and $v_{4 n+4}$, and let $B$ be the set consisting of all vertices of $O_{n} \sharp Y$ that correspond to the fundamental cycles containing the edges $v_{3} u_{1}, v_{5} u_{2}, \ldots, v_{4 n+3} u_{2 n+1}$. Let $C=V\left(O_{n} \sharp T\right)-(A \cup B)$. The subgraph of $O_{n} \sharp Y$ induced by $A$ is a complete graph on $n$ vertices, the subgraph induced by $B$ is an edgeless graph on $2 n+1$ vertices, and the subgraph induced by $C$ is a complete graph on $n+1$ vertices. It is not difficult to see that every vertex from $A$ is adjacent to every vertex from $B \cup C$ and every vertex from $C$ is adjacent to precisely four vertices from $B$. By the König-Hall Theorem [10], the subgraph induced by $B \cup C$ contains a matching $M_{1}$ of size $n+1$. Let $B^{\prime}$ be the set of vertices from $B$ not covered by $M_{1}$. The subgraph induced by $A \cup B^{\prime}$ contains a perfect matching $M_{2}$, by the König-Hall Theorem again. Clearly, $M_{1} \cup M_{2}$ is a perfect matching of $O_{n} \sharp Y$.
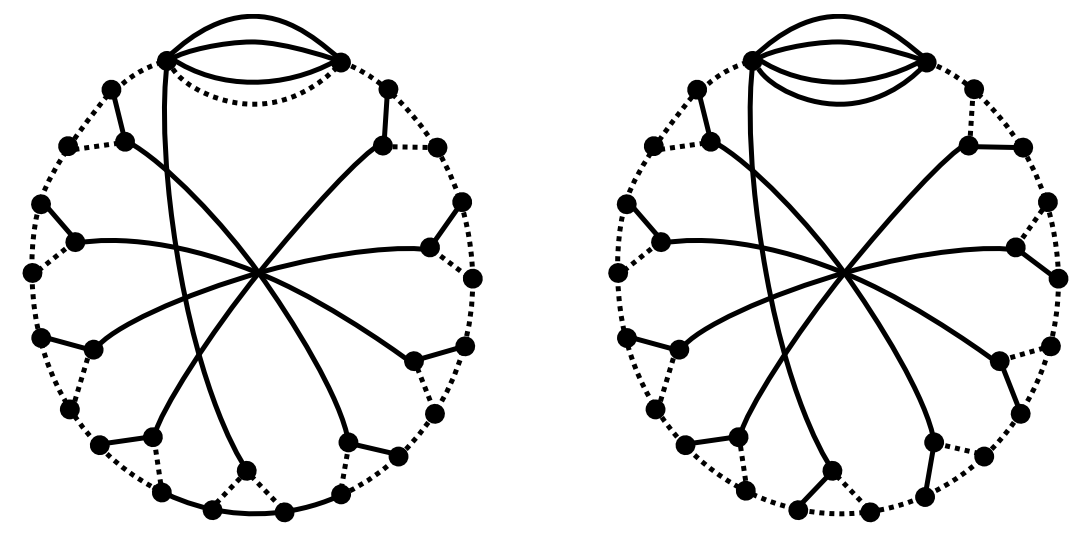

Figure 3: Spanning trees of the graph $H_{4}$ (dashed): $X$ (left) and $Y$ (right)

Theorem 12. For every positive integer $n$ there exists a graph 3-edge-connected $H_{n}$ such that

$$
\max _{T} \nu\left(H_{n} \sharp T\right)-\min _{T} \nu\left(H_{n} \sharp T\right)=n
$$

where $T$ ranges over all spanning trees of $H_{n}$. 
Proof. For each $n \geqslant 1$ set $H_{n}=O_{2 n}$; clearly, $H_{n}$ is 3-edge-connected. Theorem 1 and Proposition 10 imply that for the spanning tree $X$ described in the proof of Proposition 10 we have

$$
\min _{T} \nu\left(H_{n} \sharp T\right)=\nu\left(O_{2 n} \sharp X\right)=\gamma_{M}\left(O_{2 n}\right)=\lfloor(2 n+1) / 2\rfloor=n .
$$

On the other hand, the spanning tree $Y$ from Proposition 11 gives

$$
\max _{T} \nu\left(H_{n} \sharp T\right)=\nu\left(O_{2 n} \sharp Y\right)=2 n \text {. }
$$

The result follows.

\section{Concluding remarks}

1. The proof of Theorem 3 and polynomial-time algorithms for determining the maximum genus of a graph of Furst et al. [2] and Glukhov [4] lead to a polynomial-time algorithm for the following problem.

\section{PROBLEM: Cycle basis matching problem}

INSTANCE: Connected graph $G$, integer $k$

TASK: $\quad$ Find a cycle basis $\mathcal{B}$ of $G$ such that $\nu(G, \mathcal{B})=k$, or output 'NO' if such a basis does not exist.

Indeed, both maximum genus algorithms output a Xuong tree $T$ of a graph $G$ attaining the minimum in Theorem 1 . The bases $\mathcal{B}_{i}$ defined for $i=0, \ldots,\lfloor\xi(G) / 2\rfloor$ by equation (6) in the proof of Theorem 3 have the property that $\nu\left(J\left(G, \mathcal{B}_{i}\right)\right)=\gamma_{M}(G)+i$ for each $i$. It follows that if $k$ is between $\gamma_{M}(G)$ and $\lfloor\beta(G) / 2\rfloor$ the algorithm can output one of the bases $\mathcal{B}_{i}$ and otherwise it outputs 'NO.'

2. In Theorem 3 we have shown that for every graph $G$ the matching numbers $\nu(J(G, \mathcal{B}))$ fill in the whole interval between $\gamma_{M}(G)$ and $\lfloor\beta(G) / 2\rfloor$. This is no longer true if the bases restrict to fundamental cycles: as shown in Theorem 9 , for every integer $n$ there exists a 2-edge-connected graph $G_{n}$ such that the range of values of the matching numbers of $G_{n} \sharp T$ contains a gap of size $n$. As there can be no gap if a graph is 4-edge-connected, by Corollary 4 , the following problem suggests itself.

Problem. Does there exist, for any given $n \geqslant 1$, a 3-edge-connected graph $G$ such that the range of matching numbers of $G \sharp T$ contains a gap of size $n$ ?

\section{Acknowledgements}

Research reported in this paper was partially supported by research grants APVV-022310, VEGA 1/1005/12, and by APVV project ESF-EC-0009-10 within the EUROCORES Programme EUROGIGA (project GReGAS) of the European Science Foundation. 


\section{References}

[1] J. Chen, S. P. Kanchi, and A. Kanevsky. On the complexity of graph embeddings. In Algorithms and Data Structures', volume 709 of Lecture Notes in Comput. Sci., pages 234245, Springer-Verlag, Berlin, 1993 (extended abstract).

[2] M. L. Furst, J. L. Gross, and L. A. McGeoch. Finding a maximum-genus embedding. J. Assoc. Comput. Mach. 35: 523-534, 1988.

[3] H. Fu, M. Škoviera, and M. Tsai. The maximum genus, matchings and the cycle space of a graph. Czechoslovak Math. J. 48: 329-339, 1998.

[4] A. D. Glukhov. A contribution to the theory of maximum genus of a graph. In Structure and Topological Properties of Graphs, Inst. Mat. Akad. Nauk Ukrain. SSR, Kiev, 1981, 15-29 (in Russian).

[5] A. D. Glukhov. The maximum genus of planar graphs. Ukrainian Math. J. 34: 97-99, 1982 (in Russian).

[6] J. L. Gross and T. W. Tucker. Topological Graph Theory, Wiley, New York, 1987.

[7] M. Jungerman. A characterization of upper-embeddable graphs. Trans. Amer. Math. Soc. 241:401-406, 1978.

[8] N. P. Khomenko and A. D. Glukhov. Single-component 2-cell embeddings and maximum genus of a graph. In Some topological and combinatorial properties of graphs, Inst. Mat. Akad. Nauk Ukrain. SSR, Kiev, 1980, 5-23 (in Russian).

[9] N. P. Khomenko, N. A. Ostroverkhy, and V. A. Kuzmenko. The maximum genus of a graph In $\phi$-Transformations of Graphs, Inst. Mat. Akad. Nauk Ukrain. SSR, Kiev, 1973, 180-207 (in Ukrainian with English summary).

[10] L. Lovász and M. D. Plummer. Matching Theory, North-Holland, Amsterdam, 1986.

[11] L. Nebeský L. A new characterization of the maximum genus of a graph. Czechoslovak Math. J. 31:604-613, 1981.

[12] L. Nebeský L. Characterizing the maximum genus of a connected graph. Czechoslovak Math. J. 43:177-183, 1993.

[13] E. A. Nordhaus, B. M. Stewart, and A. T. White. On the maximum genus of a graph. J. Combinatorial Theory Ser. B 11:258-267, 1971.

[14] H. Ren, H. T.Zhao, H. L. Li. Fundamental cycles and graph embeddings. Sci. China Ser. A 52:1920-1926, 2009.

[15] R. D. Ringeisen. Survey of results on the maximum genus of a graph. J. Graph Theory $3: 1-13,1979$.

[16] J. Šráň and M. Škoviera. Characterization of the maximum genus of a signed graph. J. Combinatorial Theory Ser. B 52:124-146, 1991.

[17] N. H. Xuong. How to determine the maximum genus of a graph. J. Combinatorial Theory Ser. B 26:217-225, 1979.

[18] N. H. Xuong. Upper-embeddable graphs and related topics. J. Combinatorial Theory Ser. B 26:226-232, 1979 . 